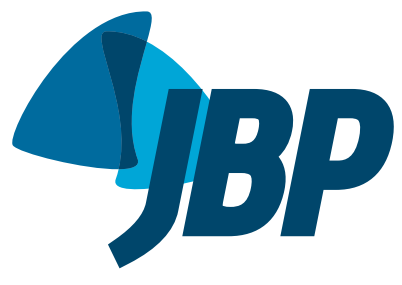

\title{
Omalizumab in patients with severe uncontrolled asthma: well-defined eligibility criteria to promote asthma control
}

\author{
Regina Maria de Carvalho-Pinto', Rosana Câmara Agondi², \\ Pedro Giavina-Bianchi ${ }^{2}$, Alberto Cukier ${ }^{1}$, Rafael Stelmach ${ }^{1}$
}

\section{TO THE EDITOR:}

After more than a decade of omalizumab being widely used in the treatment of asthma, the Brazilian National Commission for the Incorporation of Technologies stated its opposition to the incorporation of omalizumab use within the scope of the Unified Health Care System of Brazil. (1) That ruling runs contrary to expert opinion that the drug should be made available to a specific group of patients with severe uncontrolled asthma, selected according to eligibility criteria that are well defined in clinical protocols.

Here, we report the results of omalizumab administration in 12 patients with severe asthma, selected according to the strict eligibility criteria presented in Chart 1 . Nine of those patients met the criterion of lack of asthma control with appropriate treatment, and 3 met the criterion of the need for continuous doses of oral corticosteroids to maintain asthma control. Of the 12 patients evaluated, $8(70 \%)$ were female. The mean age at the initiation of treatment was $45.36 \pm 15.19$ years. The baseline FEV was $1.72 \pm 0.53 \mathrm{~L}(56.5 \pm 12.6 \%$ predicted $)$, with no change after omalizumab administration. At enrollment in the study, the patients were using a mean inhaled corticosteroid dose of 2,318.18 $\pm 844.7 \mu \mathrm{g} /$ day, and 7 patients $(63 \%)$ were using oral corticosteroid chronically, at a dose of $2.5-40 \mathrm{mg} /$ day. The mean monthly dose of omalizumab was $504.54 \pm 316.58 \mathrm{mg}$.

During the study period, omalizumab was discontinued in 1 patient, because of fainting and a rash, which were probably associated with the use of the medication. The asthma control scores of the 11 patients who completed the recommended 16 weeks of treatment are shown in Figure 1. Six of those patients had an excellent response, with evident improvements in their scores. One of those patients had ventricular tachycardia as a side effect of $\beta_{2}$ agonist use and was dependent on the use of corticosteroids, and that patient maintained control at the end of the 16-week follow-up period without the use of the $\beta_{2}$ agonist. Another 3 patients (of the 6 who clearly benefited from the treatment with omalizumab) were chronic corticosteroid users. Among those 3 patients, the corticosteroid was discontinued in 1 , whereas the dose of corticosteroid was reduced in 1 and maintained in 1 .

Two patients showed no improvement after 16 weeks of treatment, at which point the omalizumab was discontinued. In 3 patients, the response was considered partial. In 2 patients, the omalizumab was discontinued after 32 weeks, because of the occurrence of exacerbations. In 1 patient, the decision was made to continue the treatment with omalizumab. In summary, in $58 \%$ of the patients selected, we maintained the administration of omalizumab. When we used the asthma control questionnaire, defining a half-a-point variation in the score as clinically significant, the response was classified as good in $64 \%$ of the patients, compared with $73 \%$ of those when we used the asthma control test, defining a 3-point variation as clinically significant. Of the 12 patients in our sample, 8 presented a good response to omalizumab, regardless of the method employed to evaluate that response.

The decision to carry out this pilot analysis was made by the Pharmacy Board of the University of São Paulo School of Medicine Hospital das Clínicas, in 2010. At that time, there was only one nationally published study demonstrating that IgE blockade was safe in patients with asthma or allergic rhinitis caused by helminth infection. (2) However, in a multicenter study conducted in Brazil and published in 2012, Rubin et al. ${ }^{(3)}$ evaluated the use of omalizumab as an add-on therapy in patients with moderate allergic asthma that was not controlled despite treatment with the combination of long-acting bronchodilators and inhaled corticosteroids (fluticasone $\geq 500 \mu \mathrm{g} /$ day or equivalent). The authors reported improvement in asthma control and in the overall perception of efficacy among those patients. We started selecting patients in 2012, when the funds were made available in the annual budget of the institution. We estimated that it would take 1 year to conclude the analysis of 12 patients. However, because we strictly adhered to the pre-established criteria, it took nearly 3 years. Our experience was similar to that recently reported by the Australian Department of Health Subcommittee on Pharmaceutical Use. ${ }^{(4)}$ Contrary to the initial estimate that approximately 1,000 patients per year would be included in the first 5 years of the program, only 148 and 156 patients were treated in the first and second year, respectively. ${ }^{(4)}$

Although oral corticosteroid use was not a mandatory inclusion criterion, virtually all of our patients were using oral corticosteroids (regularly or continuously), as well as high doses of inhaled corticosteroids. Our criteria correspond to those approved by the Australian Department of Health ${ }^{(4)}$ and by the National Institute for Health and Care Excellence (NICE) in the United Kingdom, ${ }^{(5)}$ bodies that rely on pharmacoeconomic evaluations to guide their decisions regarding the allocation of resources for new medications.

The substantial improvement observed in some of our patients demonstrates that omalizumab is capable 

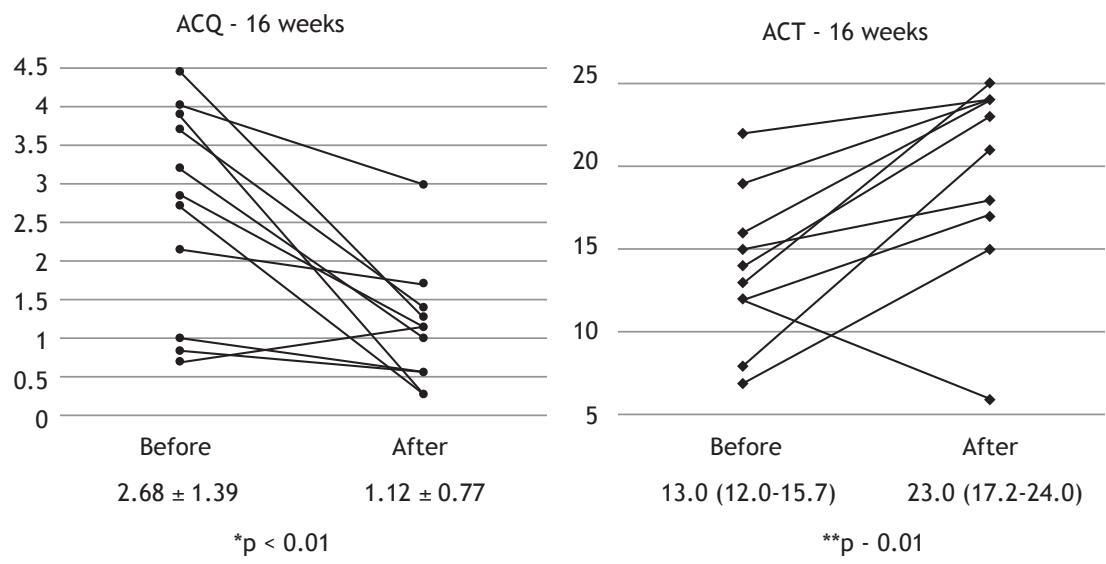

Figure 1. Evolution of asthma control scores over 16 weeks of treatment with omalizumab. ${ }^{\text {ACQ}}$ : asthma control questionnaire; and ACT: asthma control test. ${ }^{V}$ Values expressed as mean \pm SD or as median (interquartile range). *Student's t-test. **Mann-Whitney test.

Chart 1. Criteria for identifying patients eligible for treatment with omalizumab.

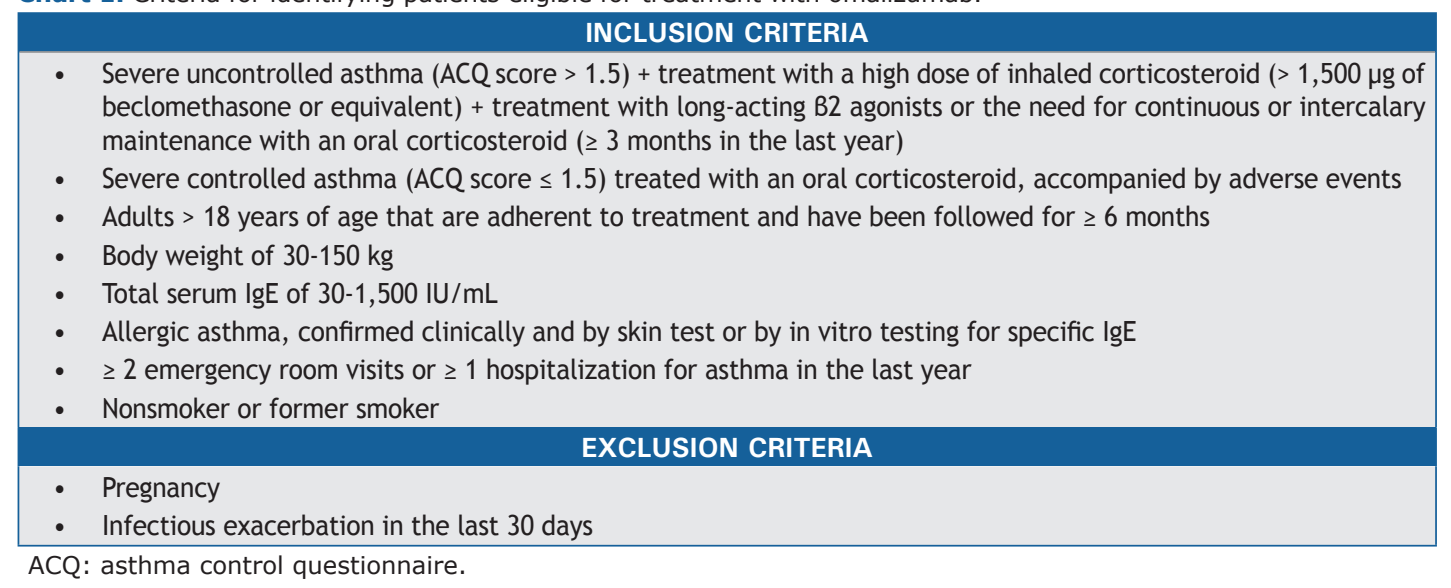

of radically altering the quality of life and the work capacity of a select portion of severe asthmatics. Similar results were taken into account in the United Kingdom in 2007, ${ }^{(3)}$ at the time of provisional approval of omalizumab, despite the unacceptable cost-effectiveness ratio, which was $>£ 30,000$ /quality-adjusted life year (QALY) gained. Not long ago, the NICE concluded that the use of the drug had become economically viable (cost-effectiveness ratio, $£ 23,200 /$ QALY gained) and limited its indication to patients with severe uncontrolled asthma who are users of oral corticosteroids and in whom the asthma remains uncontrolled even when the patients are medicated and followed according to the NICE guidelines ${ }^{(5)}$; a similar policy was adopted by the Australian Department of Health. ${ }^{(4)}$ The efficiency of those guidelines was recently confirmed in real-life studies. ${ }^{(6,7)}$

In our study, the decision to maintain the treatment not only in the responders but also in the partial responders was based on the overall evaluation of efficacy by the medical staff. That practice is supported by the literature, which demonstrates, as the best parameter of a response to omalizumab, the impression of the medical staff at week 16 (according to the package insert used in Europe, that must be considered in the decision as to whether or not to continue treatment).

Our study has certain limitations. We did not determine exactly how many patients were evaluated and how many were offered the treatment. However, that does not interfere with the main conclusion: among an estimated total of approximately 2,500 (new and follow-up) patients with difficult-to-control asthma seen over a 3-year period, the use of omalizumab resulted in substantial clinical improvement in only a small portion. Another limitation was the lack of a control group. In a study with an $n=1$ design (in which efficacy and safety are evaluated in an individual patient using a double-blind, placebo-controlled, double-blind randomized study with multiple treatment periods), Gibson et al. ${ }^{(8)}$ analyzed omalizumab administration in 12 patients with characteristics similar to those of our patients. In that study, the drug was discontinued after 12 weeks of treatment, allowing comparison between the periods with and without omalizumab treatment. The results were similar to those obtained in the present study: $50 \%$ of the patients evaluated showed a total or partial response. 
In conclusion, patients with severe asthma that remains uncontrolled despite appropriate treatment according to the available guidelines constitute only a small proportion of asthma patients and, because they are in poorer health, consume the largest share of the resources allocated. ${ }^{(9)}$ For asthma patients treated at referral centers, efforts are made to identify the factors that, duly scaled and treated, have a positive effect on the evolution of their asthma. ${ }^{(10,11)}$ The results obtained in our study, taken together with those reported in studies conducted at other centers, demonstrate that IgE blockade is effective for some patients. The application of a rigid protocol at asthma treatment centers would allow the identification of patients who might benefit from treatment with omalizumab, as opposed to prescription by litigation. ${ }^{(12)}$ Individualized and accurate medical practice, allowing equity within the system without impeding scientific progress, is the way of the future. ${ }^{(13)}$

\section{REFERENCES}

1. Brasil. Ministério da Saúde. Secretaria de Ciência, Tecnologia e Insumos estratégicos [homepage on the internet]. Brasília: o Ministério [updated 2016 Jul, cited 2017 Jan 2] Omalizumabe para o tratamento da asma grave. [Adobe Acrobat document, 110p.]. Available from: http://conitec.gov.br/images/Relatorios/2016/ Relatorio_Omalizumabe_AsmaGrave_final.pdf

2. Cruz AA, Lima F, Sarinho E, Ayre G, Martin C, Fox H, et al. Safety of anti-immunoglobulin $E$ therapy with omalizumab in allergic patients at risk of geohelminth infection. Clin Exp Allergy. 2007;37(2):197-207. https://doi.org/10.1111/j.1365-2222.2007.02650.x

3. Rubin AS, Souza-Machado A, Andrade-Lima M, Ferreira F, Honda A, Matozo TM, et al. Effect of omalizumab as add-on therapy on asthma-related quality of life in severe allergic asthma: a Brazilian study (QUALITX). J Asthma. 2012;49(3):288-93. https://doi.org/10.3 109/02770903.2012.660297

4. The Pharmaceutical Benefits Scheme (PBS) [homepage on the Internet]. Canberra: PBS [updated 2014 Jun, cited 2017 Jan 2]. Omalizumab: 24-month predicted versus actual analysis. Drug utilisation sub-committee (DUSC) [about 2 screens]. Available from: http://www.pbs.gov.au/info/industry/listing/participants/publicrelease-docs/omalizumab

5. National Institute for Health and Care Excellence (NICE) [homepage on the Internet]. London: NICE [updated 2013 Apr 24, cited 2017 Jan 2]. Omalizumab for treating severe persistent allergic asthma [about 3 screens]. Available from: http://www.nice.org.uk/guidance/ta278

6. Niven RM, Saralaya D, Chaudhuri R, Masoli M, Clifton I, Mansur AH, et al. Impact of omalizumab on treatment of severe allergic asthma in UK clinical practice: a UK multicentre observational study (the APEX II study). BMJ Open. 2016;6(8):e011857.

7. Gibson PG, Reddel H, McDonald VM, Marks G, Jenkins C, Gillman
A et al. Effectiveness and response predictors of omalizumab in a severe allergic asthma population with a high prevalence of comorbidities: the Australian Xolair Registry. Intern Med J. 2016;46(9):1054-62. https://doi.org/10.1111/imj.13166

8. Gibson PG, Taramarcaz P, McDonald V. Use of omalizumab in a severe asthma clinic. Respirology. 2007;12 Suppl 3:S35-44; discussion S45-7.

9. de Carvalho-Pinto R, Cukier A, Angelini L, Antonangelo L, Mauad T, Dolhnikoff $M$, et al. Clinical characteristics and possible phenotypes of an adult severe asthma population. Respir Med. 2012;106(1):4756. https://doi.org/10.1016/J.rmed.2011.08.013

10. Dias-Júnior SA, Reis $M$, de Carvalho-Pinto RM, Stelmach $R$, Halpern A, Cukier A. Effects of weight loss on asthma control in obese patients with severe asthma. Eur Respir J. 2014:43(5):1368-77. https://doi.org/10.1183/09031936.00053413

11. Athanazio R, Carvalho-Pinto $R$, Fernandes $F L$, Rached $S$, Rabe $K$, Cukier $A$, et al. Can severe asthmatic patients achieve asthma control? A systematic approach in patients with difficult to control asthma followed in a specialized clinic. BMC Pulm Med. 2016;16(1):153. https://doi.org/10.1186/s12890-016-0314-1

12. Stelmach $R$, Cerci Neto A, Fonseca AC, Ponte EV, Alves G, AraujoCosta IN, et al. A workshop on asthma management programs and centers in Brazil: reviewing and explaining concepts. J Bras Pneumol. 2015;41(1):3-15. https://doi.org/10.1590/S180637132015000100002

13. Sumino K, Israel E. Precision Medicine. Personalizing guidelines to the provider as well as the patient: making the juice worth the squeeze. Am J Respir Crit Care Med. 2015;191(12):1345-6. https:// doi.org/10.1164/rccm.201504-0809ED 